\title{
Sistema Experto para Diagnóstico de Plagas Insectiles de Maíz (Zea mays L.) en Centro América1
}

\author{
Francisco Leopoldo Merino Cisneros ${ }^{2}$
}

\section{COMPENDIO}

Se construyó un sistema experto para el diagnóstico de plagas insectiles de maíz en Centro América, con el propósito de poner a disposición el conocimiento técnico para el diagnóstico de insectos plagas, y de esta manera facilitar el manejo integrado de plagas en este cultivo. El trabajo se hizo en base al conocimiento adquirido de expertos del proyecto de manejo integrado de plagas, ubicado en el Centro Agronómico Tropical de Investigación y Enseñanza (CATIE). Se utilizó un sistema experto shell específico de diagnóstico, para su construcción. El sistema experto es capaz de diagnosticar 52 insectos plagas de maíz, por medio de seis múdulos de inferencia. Se usa como criterios: la fenología de la planta, 6rgano de la planta dañado, forma general del insecto, forma específica de insecto, daño general, daño específico, el orden del insecto y la familia del insecto. Además puede dar información adicional del insecto sobre sinonimias, nombre común, cido de vida, daño, situación de la plaga, y control. El sistema trahaja en el sistema operativo MS-DOS compatible con IBM y con el shell VP-EXPERT, una capacidad de memoria principal de $512 \mathrm{~Kb}$, y con dos disquetes de $360 \mathrm{~Kb}$ o uno de $720 \mathrm{~Kb}$, o disco duro.

Luego se realizó una evaluación de facilidad de uso del shell diseñado para la elaboración de sistemas expertos, dedicados al diagnústico de plagas insectiles. El propósito es comparar el nivel educacional del usuario al que se le facilita el manejo del shell. Se usó un diseño completo al azar con siete tratamientos y dncn observaciones. Los tratamientos fueron: secretaria, auxiliar de laboratorio, extensionista, técnico medio (ingeniero o licenciado), técnico medio que conoce de plagas (ingeniero o licenciado), master (MS) y doctor (PhD). Los resultados muestran que no hubo diferencia significativa entre el rango evaluado, a excepción de la facilidad. En consecuencia se concluyó queel shell presenta la operacionalidad en su manejo y que puede ser utilizado por cualquier nivel educacional dentro del rango estudiado.

Por último se comparó el sistema experto con el libro guía "Las plagas invertebradas de cultivos anuales alimenticios en América Central". Se utilizó un diseño de bloques completos al azar con dos tratamientos y 18 repeticiones, cada repetición fue una persona. Los resultados demuestran la superioridad del sistema experto sobre el libro guía; estadísticamente tiene más aciertos, requiere menos tiempo en dar respuesta y la relación aciertos/tiempo es mayor.

Palabras claves adicionales: diagnóstico, sistema experto.

\section{INTRODUCCION}

Las decisiones que el agricultor toma con respecto al agroecosistema maíz y específicamente relacionado con el subsistema plagas insectiles están intluenciadas por varios factores. Entre ellos se encuentran las percepciones del agricultor sobre las plagas y sus daños. su nivel de conocimiento de los hechos y sus valores personales.

\begin{abstract}
${ }^{3}$
An expert system was developed to diagnose maize insect pests in Central America. This system was established ta improve diagnostic procedures for insect pests and to facilitate the use of integrated pest management systems in maize. This work was based on acquired knowledge by experts in integrated pest management based at the Centro Agronómico Tropical de Investigación y Enseñanza (CATIE). A shell expert system specific for diagnostics was used. This expert system is capable of diagnosing 52 maize insects usingsix inference modules. Criterion used were: plant phenology, damaged organ ofthe plant, general form of the insect, specific form of the insect, general damage, specific damage, and order and family of the insecto In addition, this systemcan provide information on insectsynonyms, common names, life cycle, damage, pest location, and possible control. The system works with IBM compatible MS-DOS, employs the VP-EXPERT shell, requires $512 \mathrm{~Kb}$ of $\mathrm{RAM}$, and either two $360 \mathrm{~Kb}$ or one $720 \mathrm{~Kb}$ disk drives, or a hard disk.
\end{abstract}

An evaluation ofthe ease of use of the shell dedicated to diagnostics for insect pests was performed. The purpose was to compare the educationallevel of the user with the ease which the shell was used. A completely randomized design was employed with seven treatments and five observations. The treatments were: secretaries, laboratory support staff, extension agents, bachelor level personnel, M.S., and PhD. level persons. Tbe results indicated that no significant differences within the range evaluated, excluding facility of use. It was concluded that the shell presents operationalilty that can be used by various persons at different education le veis within the studied rangc.

The expert system was also compared with a book key ('Las plagas invertebradas de cultivos anuales alimenticios en America Central'). A randomized complete block design was used with two treatments and 18 replications, each replication being one persono The results dcmonstrated that the expert system was superior to the book key and that the expcrt system required much less time to obtain an answer.
La percepción del agricultor sobre la importancia de una plaga y el daño relacionado con ésta, afecta las

1 Trabajo presentado en la XXXVI Reunión Anual del PCCMCA, San Salvador, El Salvador, marzo 1990.

2 Técnico del Proyecto Manejo Integrado de Plagas CA TIE/CENTA

${ }^{3} \mathrm{El}$ abstract es traducción del compendio.

Publicado en Agronomía Mesoamericana, Vol. 2 (1991). 
decisiones sobre el método y el nivel de control de esa plaga en particular. Sin embargo, no todas las plagas ni sus daños son correctamente percibido por los agricultores, para quienes es a veces difícil hacer una asociación correcta entre el daño observado en el cultivo y la plaga que lo está causando. Esto ocasionalmente puede conducir a la conclusión errada de que un daño es causado por un insecto benéfico observado con frecuencia. Estos errores a veces conducen a decisiones incorrectas sobre métodos de control resultando a veces en daño y costos mayores.

Otro factor que influye en la toma de decisiones del agricultor sobre el manejo de las plagas es la acción de las casas comerciales. Por medio de propaganda y visitas a los agricultores, las casas comerciales tratan de influir la decisión al uso de productos que ellos venden. También, las instituciones de crédito a menudo requieren el uso profiláctico de pesticidas como una manera de asegurar la inversión contra las pérdidas en la producción causadas por plagas.

La visita constante de los extensionistas, llevando técnicas y consejos al agricultor, le ayuda al agricultor en la toma de decisiones sobre el manejo de las plagas. Sin embargo, el extensionista generalmente lleva como primera herramienta los plaguicidas, que no siempre son la opción más adecuada. Una razón para esto es, que el extensionista generalmente entra tarde como actor en la planificación de la finca; después que ya se presentó el problema. También los plaguicidas son la herramienta que mejor conoce y confía el extensionista de la región centroamericana.

La información y el conocimiento que el agricultor utiliza para tomar decisiones está influenciado por todas las personas relacionadas con él, por el problema de plagas, y por toda la propaganda a que él está sometido.

Mayor información y conocimiento sobre las plagas sería la base fundamental para ayudar al agricultor a tener una visión más amplia de alternativas más eficientes- de los métodos de control. Esta información permitiría al agricultor tomar decisiones de manejo de plagas insectiles, desde los puntos de vista del diagnóstico, manejo, y decisión económica, en lugar de depender en un solo método de control como los plaguicidas.

La obtención de la información necesaria para resolver un problema en un momento oportuno es difícil. La información práctica se encuentra principalmente en dos fuentes: la primera en el conocimiento público, que es el que aparece en los libros, publicaciones especializadas, revistas, etc., la segunda en el conocimiento de toda persona que trabaja en un campo concreto. La dificultad en la obtención de esta segunda fuente de información es no estar disponible en el momento oportuno y necesario. A veces los agricultores y extensionistas necesitan mucho más tiempo de lo disponible para diagnosticar el problema y encontrar la solución por estas formas.
El conocimiento privado es el resultado de la larga experiencia que tiene el experto en ese campo. El experto ha sacado sus propias conclusiones y las generaliza y aplica segón su criterio. Este tipo de conocimiento solo se puede alcanzar tras largos años de práctica. La accesibilidad a esta información de esta manera también es difícil, ya que el agricultor tiene acceso limitado al extensionista y el extensionista no tiene acceso a los expertos cuando es necesario. Por lo tanto a menudo se recomienda el uso de químicos, por la fácil disponibilidad del producto y del consejo, aunque muchas veces es incorrecto.

Sin lugar a duda, el disponer de la información adecuada, que provenga del conocimiento público o privado, ayudaría a optimizar la producción de maíz, bajar los costos de producción, y facilitar la toma de decisiones en el momento oportuno. Por lo tanto, deberían de existir maneras de poner el conocimiento de manejo integrado de plagas a disposición de los responsables de la divulgación de información, tales como extensionistas e investigadores. De esta manera, mejoraría la información que recibe el agricultor y la toma de decisiones que él hace.

En los años recientes, las microcomputadoras se han convertido en una herramienta que facilita el desarrollo de sistemas para ayudar a tomar decisiones. Los programas de computación son la base para el desarrollo de este tipo de sistemas integrados de manejo de información. Los sistemas expertos son una forma de manejar integradamente la información, para ayudar a tomar decisiones a problemas planteados en campos específicos.

Estos sistemas expertos son ó presentan una alternativa potencial debido a la necesidad de obtener y hacer más accesible los criterios de los expertos y mejor información sobre el manejo integrado de plagas, por ser escasos actualmente en América Central. Los sistemas expertos son una herramienta que podrá ser utilizada cuando se necesite la opinión de un experto o información sobre un problema específico. Al mismo tiempo, la información requerida estará disponible en el momento que se solicite, y baj? un criterio altamente especializado, sin la presencia física del experto o el material bibliográfico.

El objetivo de este estudio fue el desarrollo de un sistema experto para el diagnóstico de plagas insectiles de maíz en América Central. Se desarrolló un sistema experto tipo shell que da las bases para hacer sistemas expertos de diagnóstico de plagas insectiles en diferentes cultivos. En el trabajo también se hizo prueba de la facilidad de uso del shell, así como una comparación de capacidad de diagnóstico, entre el sistema experto de plagas insectiles de maíz y un manual (King y Saunders, 1984) frecuentemente usado para tal propósito en América Central.

\section{REVISION DE LITERATURA}

Las plagas afectan la producción de los cultivos 
alimenticios en América CentJ'al y los agricultores se ven afectados por la reducción en el rendimiento (King y Saunders 1984). En los Estados Unidos se pierden el treinta y siete porciento de la producción potencial de los cultivos debido a plagas (Hom, 1988).

Para evitar las pérdidas en los cultivos por plagas, se toman medidas de control que aumentan los costos de producción. En Costa Rica los costos asociados con el control de plagas de maíz representa el 23\% del total de costos de producción (Hilje et al., 1987).

Para minimizar las pérdidas y hacer el menor daño posible al ambiente con el menor costo, las plagas deben de ser correctamente diagnosticadas, reconocer al temati vas de manejo y tomar decisiones de acción apropiadas (King y Saunders, 1994).

Como plaga se entiende cualquier animal, planta o microorganismo que compita con el hombre por los alimentos que él produce (King y Saunders, 1984; Hom, 1988). Las plagas insectiles pueden ser divididos en: constantes; de invasión; de hajo nivel, intermitentes o auxiliares; y de vectores (King y Saunders, 1984). Las plagas insectiles están compuestas por insectos que dañan a las plantas. En Nicaragua se identificó 15 insectos plagas de importancia en maíz (MAG/FAO/PNUD 1976). King y Saunders (1984) reportan 48 plagas insectiles del maíz en la región centroamericana. Para hacerle frente a estas plagas, el agricultor tiene que identificarlas y tomar decisiones con respecto a las alternativas de manejo (Hom, 1988).

La selección del control químico no siempre soluciona los problemas a largo plazo, a pesar de que al principio de su uso se obtienen buenos resultados, más tarde se puede producir perturbaciones en el agroecosistema. Esto resulta en su mayor uso del control químico, aumentando los costos de producción del cultivo hasta que no es rentable, (MAG/FAO/PNUD, 1976).

El manejo integrado de plagas es el uso racional e inteligente de todos los recursos disponibles, basados en la hiología y ecología de la plaga, con el propósito de bajar la densidad de ésta más allá del umbral económico, donde el daño hecho no justifique ya el costo de un esfuerzo de más acción (King y Saunders, 1984; MAG/FAO/PNUD, 1976).

Otras tácticas de control de plagas incluyen el biológico, el cultural, el físico, el microbial, el fitogenético y el químico. El control biológico aprovecha los enemigos naturales de la plaga; el control cultural utiliza prácticas agronómicas para suprimir a la plaga; el control físico altera el régimen de humedad, temperatura, o utiliza luz negra o electricidad en trampas; el microbial, control de la plaga por medio de enfermedades de los insectos plagas provocados por el hombre; el control fitogenético utiliza variedades de plantas resistentes a las plagas; y el control químico usa pesticidas químicos (Horn, 1988; MAG/FAO/PNUD 1976).
La decisión para el control de plagas está basada en la población o su daño, y los umbrales de acción. Se realiza un muestreo con el propósito de conocer la densidad aproximada de la plaga. Para medir ésto se puede estimar la presencia del insecto, o en el daño que éste causa. El umbral de acción es la densidad de la plaga a la cual el agricultor decide controlar, ésta puMe ser basada en una recomendación técnica o en la experiencia del agricultor. (King y Saunders, 1984; Hom, 1988; MAGIFAO/PNUD, 1976).

Las decisiones sobre el manejo de plagas por el hombre puede ser mejorado con el uso de sistemas computarizados que utilizan inteligencia artificial, donde un gran desafío es combinar los modelos de simulación con esquemas de recomendaciones basados en sistemas expertos (Hom, 1988).

Un sistema experto es definido como un programa inteligente de computadora que usa conocimiento e inferencia producidos para resolver problemas que son bastante difíciles y requieren significativa experiencia humana para su solución. El conocimiento necesario para sugerir o recomendar, más el procedimiento de inferencia usado, puede ser a través de la experiencia del mejor practicante en ese campo (Harmon y King, 1985; Parsage y Chignell, 1988; Weiss y Kulikonski, 1984; Chadwick y Hannah, 1987).

El impacto de la nueva tecnología en el manejo integrado de plagas se está manifestando en nuevos métodos para modelar el medio ambiente, el muestreo biológico, y el desarrollo de sistemas de apoyo para la toma de decisiones (Croft, 1985).

Las condiciones que crean la necesidad de la ayuda de la computadora para las decisiones del hombre son: la cantidad, complejidad y análisis requerido de la información, el alto control de ésta, y la necesidad de acceso rápido. Además contribuye a esto la acelerada investigación y la generación de gran cantidad de información biológica sobre la interacción plaga-hospedero, vista en el contexto de un ecosistema (Naegele et al., 1985).

Avances recientes en la tecnología de las computadoras han hecho posible el desarrollo de sistemas expertos. En la agricultura estos pueden ser útiles para resolver problemas sobre riego, problemas nutricionales y fertilización, control de malezas, control de insectos, y control de enfermedades. El sistema experto tiene la ventaja que después que es desarrollado, eleva el desempeño del trabajador promedio (Mckinion y Lemmom, 1985; Wisiol y Hesketh, 1988).

En el campo de la entomología, el sistema experto es relevante para resolver problemas de diagnóstico. Tiene potencial para integrar diversos tipos de conocimiento dentro de un programa fácil de usar que ayuda a tomar decisiones, convirtiendo al sistema experto en una herramienta extremadamente poderosa para ser usada en la investigación y extensión entomológica (Stone et al. 1986). 
En América Central aún no se ha desarrollado un sistema experto para el manejo integrado de plagas insectiles de maíz. Sin embargo, se han hecho bases de datos para ser utilizados como un sistema de referencia entomológico (Saunders, et al, 1983; Reyes y Hernández, 1987).

En la parte entomológica se han desarrollado algunas aplicaciones de sistemas expertos como: SYSTEX que es un sistema experto de diagnóstico para la identificación del insecto del género Signiphora, otro caso es el sistema integrado llamado Fl.EX para la producción de algodón en Texas (Stone et al., 1986).

\section{MATERIALES Y MÉTODOS}

La elaboración del presente trabajo se llevó a cabo en el Centro Agronómico Tropical de Investigación y Enseñanza (CATIE), Turrialba, Costa Rica.

El estudio completo está compuesto por cuatro etapas independientes pero íntimamente relacionado, los cuales fueron desarrollados por el autor con la ayuda del comité asesor.

La primera parte fue montar el comité asesor, que debido a la naturaleza del trabajo, se formó por especialistas en diferentes disciplinas, como: en entomología, computación, economía, y sistemas de producción.

La segunda parte consistió en diseñar y construir un sistema experto shell para el desarrollo de sistemas expertos de diagnóstico de plagas insectiles. En esta parte se diseñó el modelo conceptual y operativo del sistema experto shell, así como la codificación de éste en lenguaje VP-EXPERT, y además se hicieron bases de datos y de textos. En esta etapa los especialistas sugirieron la manera en que podía generalizarse el diagnóstico, así como el conocimiento mínimo que éste debía contener y manejar.

La tercera etapa de la tesis fue el desarrollo de un sistema experto para el diagnóstico de plagas insectiles de maíz en América Central. Para llevarse a cabo, hubo que apoyarse el shell elaborado en el primer trabajo, ésto redujo el trabajo de programación, lo cual permitió concentrarse en la adquisición del conocimiento sobre las plagas insectiles que atacan el maíz.

El conocimiento adquirido se obtuvo de revisión de literatura, pero principalmente de entrevistas personales con los especialistas en entomología. El conocimiento que se necesitaba era: la fenología de la planta cuando es atacada, el órgano de la planta atacado, descripción del daño general, descripción del daño específico, la forma general del insecto, la forma específica del insecto, el orden del insecto, la familia del insecto, un texto que describa al insecto en su distribución geográfica, su ciclo de vida, huéspedes, daño, situación de la plaga, y control. Con esta información se construyó el sistema experto.
Luego el sistemaexperto fue veriflcadoporel espeeiaüsta que dio el conocimiento, y fue evaluado por otros especialistas quienes rectificaron y aumentaron el conocimiento.

En la cuarta etapa se evaluó. la facilidad de uso del sistema experto shell, en donde se comparó el tiempo, los aciertos y la opinión de facilidad de los participantes. Para hacer la evaluación, se utilizó un diseño completamente al azar, en donde hubo cinco observaciones y siete tratamientos. Los tratamientos consistieron en personas con diferentes grados de edycación; secretarias, auxiliares de laboratorio, extensionistas, técnico medio (Ingeniero o Licenciado), técnico medio que conoce de plagas (Ingeniero o Licenciado), master (M.Sc.) y doctor (Ph.D).

La información de las variables se obtuvo al pasar a cada persona una guía de seguimiento, luego tomar el tiempo y los aciertos, por último se preguntaba su opinión a cerca de la facilidad que había notado.

Después de haber obtenido los datos se procedió a analizarlos y a estudiar los resultados, alcanzando al final las conclusiones.

En la quinta etapa del trabajo se realizó una comparación qel sistema experto para diagnóstico de plagas insectiles de maíz en América Central y el libro guía, "Las plagas invertebradas de los cultivos anuales alimenticios en América Central" (King y Saunders, 1984).

En los dos sistemas de diagnóstico se compararon el número de aciertos logrados al realizar doce diagnósticos con el libro guía y con el sistema experto, también se tomó como dato el tiempo en que se hicieron los diagnósticos.

Se utilizó un diseño de bloques al azar con dos tratamientos y 18 repeticiones. Las repeticiones fueron estudiantes de maestría en el área de manejo integrado de plagas, pero que no eran especialistas en entomología.

Cada repetición hizo doce diagnósticos con el libro guía y doce con el sistema experto en total veinticuatro diagnósticos, estos fueron intercambiados en cada repetición.

Luego de haber obtenido los datos, se analizaron estadísticamente y de los resultados se obtuvieron las conclusiones pertinentes.

Para llevar a cabo todo el trabajo se utilizó una microcomputadora marca HYUNDAI, modelo Super-286C, con un microprocesador Intel 80286, un disco duro de $40 \mathrm{MB}$ de un manejador de discos (drive) de $51 / 4 "$ ", un manejador de discos (drive) de 3.5", un monitor monocromático de alta resolución. una tarjeta de gráficos multivido marca ATI, y una impresora de matrices marca EPSON LX-800 de 180 cps. 


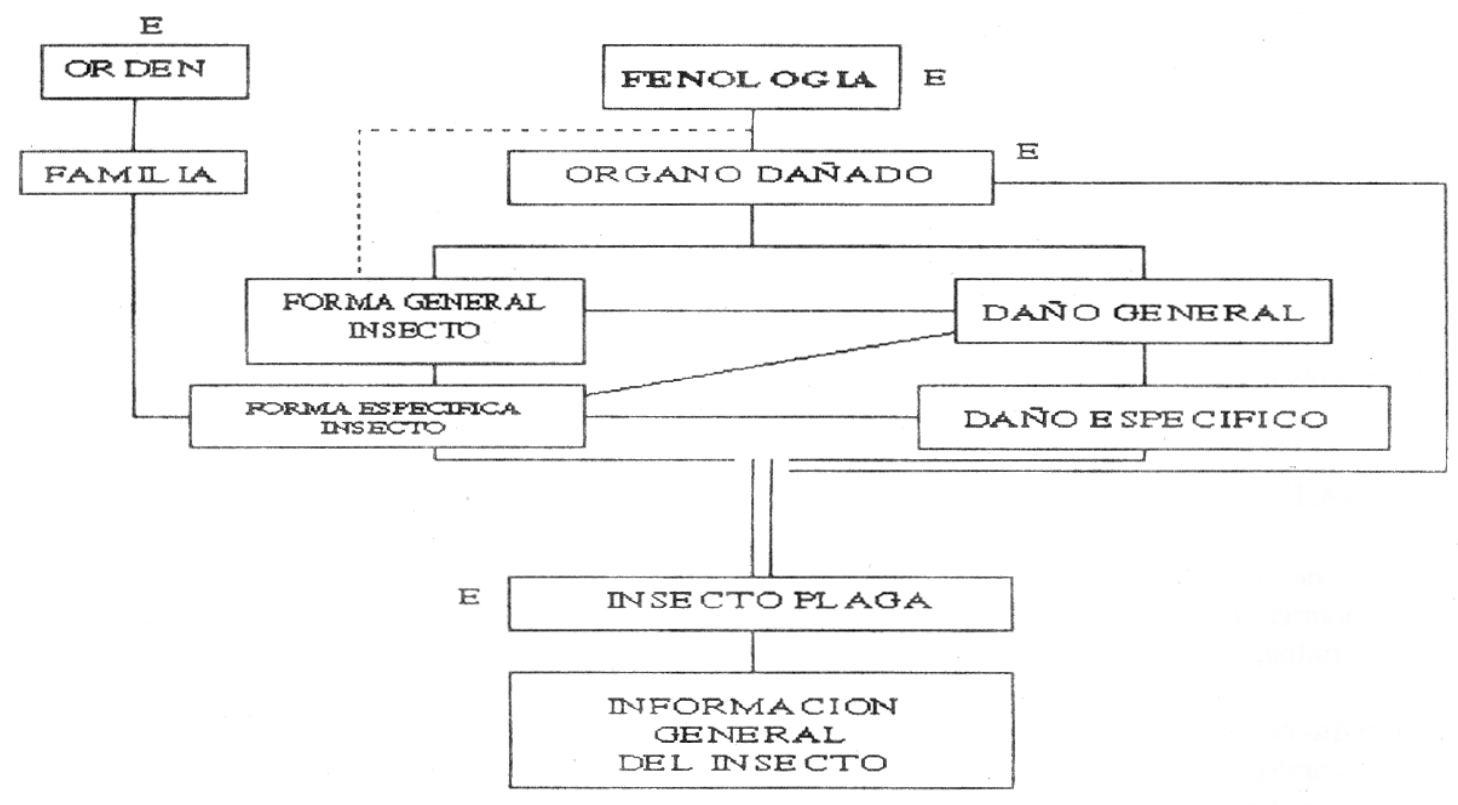

$E=$ posible estrad a para la identificación del insecto

Figura 1 Estructura del modelo conceptual que posee el Sistema Experto de diagnóstico

Cuadro 1 Principales plagas insectiles de maíz de América Central.

\begin{tabular}{|c|c|c|}
\hline Familia & Plagas & \\
\hline Acrididae & $\begin{array}{l}\text { Agrotis ipsilon } \\
\text { Chaetocnema } \mathrm{sp} \\
\text { Cyrtomenus } \mathrm{sp} \\
\text { Diatraea } \mathrm{sp} \\
\text { Feltia subterranea } \\
\text { Listronotus } \mathrm{sp} \\
\text { Oligonychus } \mathrm{sp} \\
\text { Sathrobrota } \mathrm{sp} \\
\text { Typophorus } \mathrm{sp} \\
\text { Anomala } \mathrm{sp} \\
\text { Chaetopsis } \mathrm{sp} \\
\text { Dalbulus } \mathrm{sp}\end{array}$ & $\begin{array}{l}\text { Atta sp } \\
\text { Colopterus } \mathrm{sp} \\
\text { Diabrotica porracea } \\
\text { Euetheola sp } \\
\text { Heliothis zea } \\
\text { Mocis sp } \\
\text { Pococera sp } \\
\text { Spodoptera frugiperda } \\
\text { Acheta } \mathrm{sp} \\
\text { Blissus sp } \\
\text { Conotelus } \mathrm{sp} \\
\text { Diabrotica } \mathrm{sp}\end{array}$ \\
\hline Elateridae & $\begin{array}{l}\text { Eumecosomyia sp } \\
\text { Hylemya sp } \\
\text { Mythimna sp }\end{array}$ & $\begin{array}{l}\text { Frankliniella sp } \\
\text { Metachroma sp } \\
\text { Peregrinus sp }\end{array}$ \\
\hline Romelaeidae & Sitophilus sp & \\
\hline Tettigonidae & $\begin{array}{l}\text { Acromyrmex sp } \\
\text { Cathartus } \mathrm{sp} \\
\text { Crambus } \mathrm{sp} \\
\text { Diabrotica viridula } \\
\text { Euxesta } \mathrm{sp} \\
\text { Ligyrus } \mathrm{sp} \\
\text { Neocurtilla } \mathrm{sp} \\
\text { Rhopalosiphum } \mathrm{sp} \\
\text { Tominotus } \mathrm{sp}\end{array}$ & $\begin{array}{l}\text { Araecerus sp } \\
\text { Collaria sp } \\
\text { Diabrotica balteata } \\
\text { Elasmopalpus sp } \\
\text { Geraeus sp } \\
\text { Metamasius sp } \\
\text { Phyllophaga sp } \\
\text { Solenopsis sp }\end{array}$ \\
\hline
\end{tabular}

\section{RESULTADOS}

Los resultados obtenidos en la fase de estmcturación del razonamiento del sistema experto se muestra en la Figura 1, la estructura es el modelo conceptual del sistema experto que muestra la manera lógica de identificar un insecto plaga de maíz según el especialista.

En el Cuadro 1 se muestra los 52 insectos plagas que se tomaron en cuenta en el sistema experto de diagnóstico de maíz.

Los estados fenológicos que se usaron en maíz fueron:

1) Germinando

2) Plántula

3) Planta grande

Los órganos de la planta dañados por los insectos plagas, considerados por el experto fueron:

1) Raíz

2) Tallo

3) Hojas y cogollo 4) Mazorca

Los órdenes de insectos y ácaros plagas encontrados en maíz y usados en el sistema fueron: 


$\begin{array}{ll}\text { Acarina } & \text { Homoptera } \\ \text { Coleóptera } & \text { Hymenoptera } \\ \text { Diptera } & \text { Lepidoptera } \\ \text { Hemiptera } & \text { Orthoptera } \\ \text { Thysanoptera } & \end{array}$

Las familias de insectos y ácaros plagas encontrados en maíz y usados en el sistema fueron:

$\begin{array}{ll}\text { Acrididae } & \text { Gryllotalpidae } \\ \text { Antbomyiidae } & \text { Lygaeidae } \\ \text { Antbribidac } & \text { Miridae } \\ \text { Apbidirule } & \text { Nitidulidae } \\ \text { Chrysomelidae } & \text { Noctuidae } \\ \text { Cicadellidae } & \text { Otitidae } \\ \text { Cosmopterigidae } & \text { Pyralidae } \\ \text { Cucurlionidae } & \text { Romelaidae } \\ \text { Cidnidae } & \text { Scarabaeidae } \\ \text { Delphacidae } & \text { Silvanidae } \\ \text { Elateridae } & \text { Tetracbinydae } \\ \text { Formicidae } & \text { Tettigonidae } \\ \text { Grillidae } & \text { Thripidae }\end{array}$

Las formas generales encontradas en los insectos plagas de maíz y usados en el sistema fueron:

$\begin{array}{ll}\text { Acaro } & \text { Grillo } \\ \text { Afido } & \text { Hormiga } \\ \text { Chapulín } & \text { Larva } \\ \text { Chinche } & \text { Picudo } \\ \text { Escarabajos } & \text { Saltahoja } \\ \text { Gorgojo } & \text { Trips } \\ \text { Zompopo } & \end{array}$

Las diferentes formas específicas encontradas y utilizadas por el sistema fueron 48, además de 19 daños generales y 30 daños específicos.

En los resultados obtenidos de la prueba de facilidad de uso del sistema se tienen los análisis de las variables número de aciertos, porcentaje de aciertos, tiempo de respuesta en minutos, facilidad en una escala y número de aciertos/ tiempo de respuesta.

En el cuadro 2 se observa que la variable facilidad es la única que tiene diferencias signit1cativas entre los niveles de educación. Para las otras variables el comportamiento de los niveles educacionales no fue posible detectar diferencias entre ellos.

La prueba de Tukey muestra que existe diferencias significativas entre las opiniones del Técnico medio y las secretarias extensionistas y los técnicos medios que conocen de plagas. Para los Master, Doctores y auxiliares de laboratorio no se encontró diferencia significativa. Esto se observa en el cuadro 3.
Cuadro 2 Probabilidades y significancias de las diferencias entre los niveles de educación para las variables analizadas.

\begin{tabular}{lccc}
\hline Variable & F calculada & Pr $>$ F & Significancia \\
\hline Número de aciertos & 0.90 & 0.5137 & NS \\
Porcentaje de aciertos & 0.90 & 0.5137 & NS \\
Tiempo de respuesta & 0.45 & 0.8343 & NS \\
Facilidad & 5.39 & 0.0012 & $* *$ \\
No aciertos / Tpo resp. & 0.29 & 0.8790 & NS \\
\hline
\end{tabular}

$\mathrm{NS}=$ No significativa

$* *$ = Altamente significativa

Cuadro 3 Comparación de medias de facilidad, con una prueba de Tukey al $5 \%$ de probabilidades de error.

\begin{tabular}{lll}
\hline Tratamicnto & Media & Grupo \\
\hline Técnico medio (nngeniero o Licenciado) & 2.0 & $\mathrm{~A}$ \\
Master (MSc) & 1.6 & $\mathrm{AB}$ \\
Auxiliar de laboratorio & 1.4 & $\mathrm{AB}$ \\
Doctor (PhD) & 1.4 & $\mathrm{AB}$ \\
Secretaria & 1.2 & $\mathrm{~B}$ \\
Extensionista & 1.0 & $\mathrm{~B}$ \\
Técnico medio conoce plagas. & & \\
(Ingeniero o Licenciado) & 1.0 & $\mathrm{~B}$ \\
\hline
\end{tabular}

1 = Muy Fácil 2 = Fácil

Cuadro 4 Probabilidades y significancias de las diferencias entre in: niveles de educación para las variables analizadas.

\begin{tabular}{lccc}
\hline Variables & F calculada & Pr $>$ F & Significancia \\
\hline Porcentaje de aciertos & 12.75 & 0.0024 & $* *$ \\
Tiempo de respuesta & 41.61 & 0.0001 & $* *$ \\
No aciertos/Tpo. resp. & 55.39 & 0.0001 & $* *$ \\
\hline
\end{tabular}

** = Altamente significativa

Cuadro 5 Comparación de medias del sistema experto y libro guía con la prueba de Tukey al 5\%, para diferentes variables.

\begin{tabular}{lcc}
\hline Variables & Libro Guía & Sistema Experto \\
\hline Porcentaje de aciertos & $75,93 \mathrm{~B}$ & $92,59 \mathrm{~A}$ \\
Tiempo en minutos & $38,76 \mathrm{~A}$ & $19,43 \mathrm{~B}$ \\
Aciertos/tiempo & $0,26 \mathrm{~B}$ & $0,60 \mathrm{~A}$ \\
\hline
\end{tabular}

Letras iguales no son significativamente diferentes

El análisis de varianza de la comparación del sistema experto y el libro guía, los factores en estudio mostraron los siguientes resultados: en el Cuadro 4 se observa que hubo diferencias significativas en las variables: porcent.ajede aciertos, tiempo en minutos y relación aciertos/tiempo.

La prueba de medias muestra que exist.e diferencia significativa entre el sistema experto y el libro guía (ver Cuadro 5).

En las figuras 2 y 3 se muestra que el sistema experto es mayor en porcentaje de aciertos y la relación acier- 
tos/tiempo, que el libro guía. En la figura 4 se observa que el tiempo del sistema experto es menor que el libro guía. Además se encontró que el coeficiente de variación del sistema experto es menor que el libro guía, como lo muestra la Figura 5.

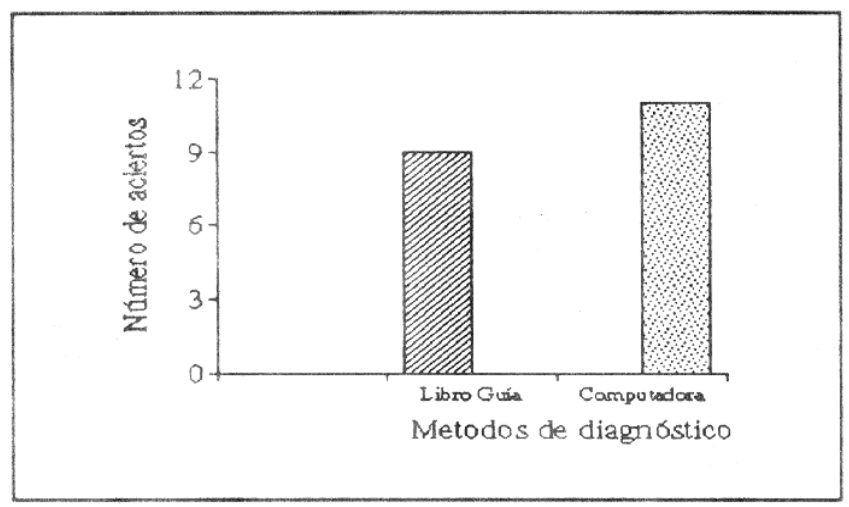

Figura 2 Comparación del número de aciertos para dos métodos de diagnóstico

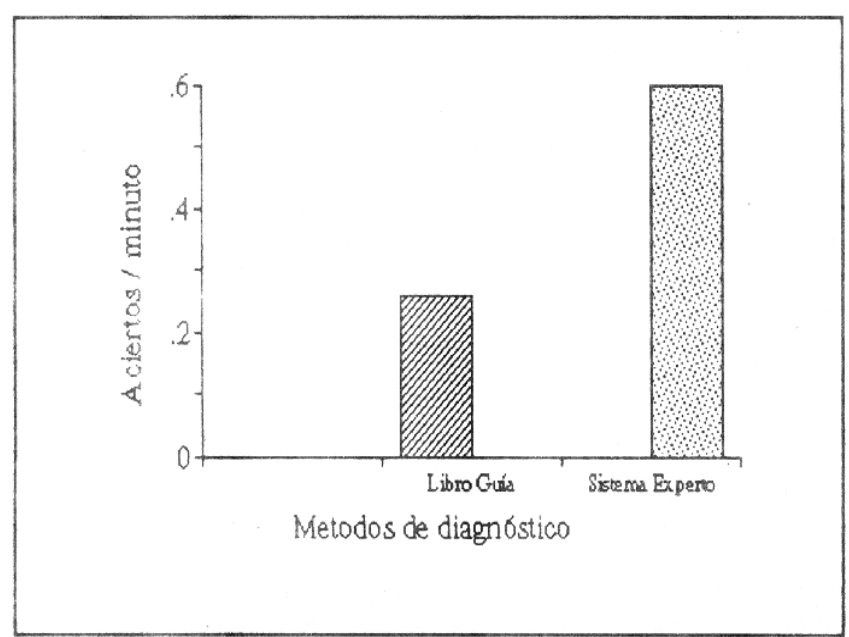

Figura 3 Comparación del tiempo de respuesta para dos métodos de diagnóstico.

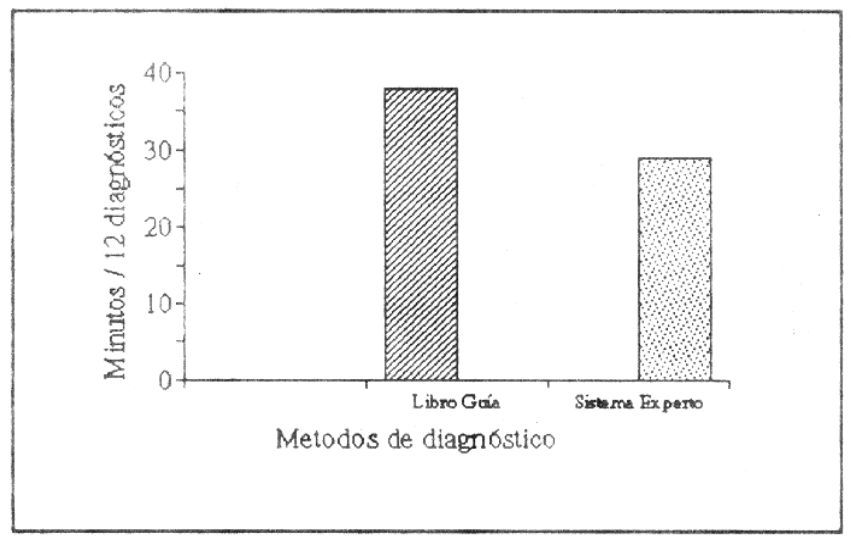

figura 4 Comparación del tiempo de respuesta para dos métodos de diagnóstico.

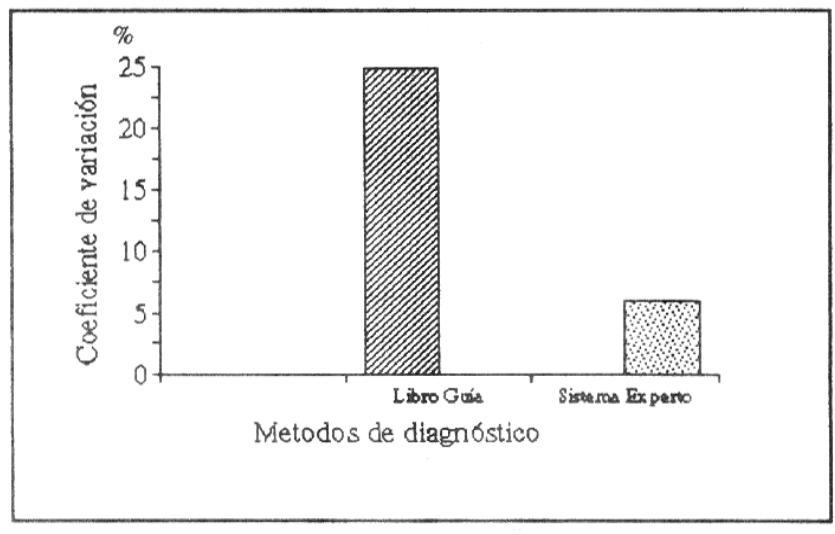

Figura 5 Coeficiente de variación de dos métodos de diagnóstico.

\section{DISCUSIÓN}

El sistema experto para el diagnóstico de plagas insectiles en maíz, fue realizado para obtener el conocimiento de todas las plagas insectiles propuestas. El mayor trabajo se concentró en la adquisición del conocimiento, específicamente en las relaciones de las características de cada plaga, debido a las diferentes formas de interpretación que puede darse a cada descripción, por lo que se tuvo que ser lo más claro y universal.

También la metodología de adquisición de la información hubo que modificarla constantemente, tratando de sacar la mayor cantidad de información del experto en el menor tiempo posible, ya que los especialistas son personas muy ocupadas, y se trata de quitarles el menor tiempo, para estar consultando con más frecuencia.

La codificación es relativamente fácil un vez que se tiene el material condensado. Los códigos pueden ser colocados sin problemas y de la manera que se quiera, sin embargo se observó que era necesario tomar algún patrón para facilit'lf el manejo de los códigos.

La representación del conocimiento se limitó a construir las bases de hechos y códigos con el administrador de base de datos DBASE IV, y hacer los archivos ASCII con el procesador de palabras WORD. El uso del shell simplificó este paso.

La verificación como la validación jugaron un papel importante en la calidad y precisión de la información, ya que la observación del comportamiento del sistema, por parte de los expertos, condujo a eliminar errores que el sistema poseía.

Sin duda el uso del "sistema experto shell", así como la correcta selección de los especialistas y la metodología, ayudan a facilitar la construcción de sistemas expertos para la identificación de plagas insectiles.

Para la prueba de facilidad de uso los análisis de todas 
las variables con excepción de facilidad no mostraron diferencias significativas. Esto es debido a que la estructura del sbell ayuda a que el usuario independientemente de su nivel educacional sea capaz de poder seguir una secuencia de preguntas y respuestaspJanteadas por el programa. Además es posible por la interacción que tiene el shell con el usuario a través de la utilización de menós, que ante una pregunta planteada, muestra todas las posibles respuestas que el sistema experto es capaz de responder por lo que se hace difícil cometer un error. En consecuencia los aciertos y el tiempo, son similares para los diferentes niveles educacionales.

La variable facilidades subjetiva, y dependió del criterio personal de cada una de las personas que participó en el experimento. Por eso es razonable pensar que las diferencias significativas que hubo tanto en el nivel educacional y entre las repeticiones, es debido a las diferencias que hay entre cada participante. Sin embargo la diferencia se encontró dentro de las respuestas fácil (calificación 2.0) y muy fácil (calificación 1.0), y solamente los técnicos medios (Ingeniero o Licenciado) encontraron fácil el uso del shell, el resto de los niveles educacionales 10 hallaron muy fácil o indecisos entre. fácil y muy fácil. Generalmente se puede asumir que el uso del shell es fácil y con un poco de práctica llegaría a ser muy fácil para todos los niveles educacionales, ya que no hubo opiniones en contra del uso de sistema experto.

En el cuadro 5 es notorio observar que la evidencia experimental muestra ventaja del sistema experto sobre el libro guía. En el caso del porcentaje de aciertos el sistema experto tiene un $92.59 \%$ contra el libro guía que posee un $75.93 \%$. Esto es particularmente interesante debido a que uno de los participantes de la creación del libro guía es la misma persona que proporcionó su conocimiento al sistema experto. Lo que hace pensar que no debería haber diferencia entre los dos métodos ya que el conocimiento es similar, y si agregamos que el sistema experto tuvo como base el libro guía, ésto lo comprobaría. Sin embargo el libro guía tiene una diferencia de cinco años de publicación con respecto al desarrollo del sistema experto. La importancia radica, en la acumulación de más experiencia por parte del experto, así como asimilación y comprensión de las dificultades que el libro guía presenta y que no se cometieron al crear el sistema experto. Por otro lado, además del conocimiento del experto principal se agregó conocimiento adicional de dos expertos más, que también verificaron el conocimiento del experto principal. Por lo tanto el conocimiento que posee el sistema experto está presentado de forma más clara y fácil de entender que el libro guía. Lo que es evidente con los resultados obtenidos.

El tiempo es otra variable en donde se observa diferencia significativa entre los tratamientos. La media para el sistema experto es de 19.43 minutos siendo menor que el libro guía que tiene una media de 38.76. La comparación entre el sistema experto y el libro guía con respecto al tiempo tiene sentido si se piensa como una comparación mecánica de búsqueda de la información en donde la computadora y el sistema experto se conjugan para encontrar las respuestas más rápidamente que si se hiciera manualmente como en el caso dellibro guía. Esto ha sido probado ya con el uso de bases de datos que han mostrado sus bondades al facilitar la búsqueda de información de forma muy rápida.

La relación entre el número de aciertos y el tiempo es importante ya que de esta manera se puede observar con mayor claridad la ventaja del sistema experto sobre el libro guía. Se pudo encontrar un sistema rápido pero con pocos aciertos o al contrario, que sin la relación aciertos y tiempo, se dificultaría explicarla. Sin embargo, está clara la ventaja del sistema experto al observar la media de 0.60 contra 0.26 aciertos/minuto del libro guía que representa una eficiencia de $230.77 \%$ del sistema experto. Otra observación importante es el Coeficiente de variación en donde el sistema experto tiene un valor de $6.1 \%$ y el libro guía $24.7 \%$ mostrando una tendencia a las respuestas más lejanas de la media, en el caso del libro guía y mucho más cercanas de la media, en el caso del sistema experto. Una posible explicación a este fenómeno puede darse en la especificidad del sistema experto ya que solo posee conocimiento de plagas insectiles de maíz, y el libro guía posee conocimiento de plagas insectiles de más cultivos permitiendo que el usuario tenga más posibilidades de distracción y selección cuando hace la búsqueda. Esto no sucede con el sistema experto dando más posibilidades de acertar, bajando el coeficiente de variación.

\section{CONCLUSIONES}

1) El sistema experto de diagnóstico de plagas insectiles de maíz puede identificar 52 insectos plagas diferentes.

2) El uso del "sistema experto shell" facilitó la construcción del sistema experto de diagnóstico de plagas insectiles de maíz.

3) Basado en la observación, el uso de menús facilita la utilidad del sistema.

4) La experiencia de los expertos, disminuyó el tiempo en la adquisición del conocimiento.

5) El uso shell para hacer sistemas expertos en diagnósticos de plagas insectiles para cualquier cultivo, es independiente el nivel educacional que tengan los usuarios. dentro del rango estudiado.

6) La interacción del shell con el usuario por medio de menús facilita el aprendizaje de manejo

7) El sistema experto de diagnóstico de plagas insectiles de maíz es mejor significativamente que el uso del libro guía con respecto al porcentaje de aciertos, en tiempo de respuesta y la relación número de aciertos sobre el tiempo de respuesta. 
8) Se puede diagnosticar plagas insectiles de maíz con ecosistema experto de diagnóstico de plagas insectiles de maíz de. manera aceptable.

9) Basado en la observación, los aciertos en el diagnóstico depende de la calidad y la claridad en que están expuestos los conocimientos.

10) El tiempo que se tarda un diagnóstico depende del método de búsqueda, siendo mejores los métodos mecánicos como la computadora comparándola con el libro, especialmente cuando los volúmenes de información son grandes.

11) Existe la posibilidad de que los sistemas expertos de diagnósticos de plagas puedan servir para otros cultivos.

\section{RECOMENDACIONES}

1) El sistema experto construido debe ser mantenido, agregando conocimiento nuevo y quitando conocimiento obsoleto.

2) Se debe comparar el sistema experto con otros sistemas tradicionales de diagnóstico de plagas insectiles.

3) Se recomienda que se construyan otros sistemas similares para diferentes cultivos.

4) Hacer la prueba de facilidad de uso con otros grupos de personas, como estudiantes de las universidades, de institutos técnicos, trabajadores de campo y oficina, agricultores, etc., así como las personas estudiadas y en los países donde se utilizará el shell.

5) El sistema experto de diagnóstico de plagas insectiles de maíz debe ser probado en los diferentes países de Centro América, para validar su funcionamiento en esas condiciones.

6) Al sistema experto se le debe incorporar conocimiento específico de cada país del área centroamericana, para adaptarlo a sus necesidades.

7) Todos los métodos de diagnósticos como libros, claves, guías, base de datos, sistemas expertos, etc. deben ser evaluados antes de distribuirlos al usuario.

8) Debe darse un apoyo al desarrollo de sistemas expertos, modelos de simulación, sistema de soporte de decisiones y bases de datos, para ser usados en agricultura y ganadería.

\section{BIBLIOGRAFIA}

CHADWICK, M.; HANNAH, J. 1987. Expert system for microcomputers an introduction to artificial intelligence. E.E.U.U. TAB. 234 p.

CROFf, B.A. 1985. Integrated pest man.,ement: the agricultural- enviromental rationale. pp 712-728. In Frisbie, R.C. y Adkinsson, P.L. (eds). Integrated pest managementon majoragricultural systems. Texas A \& M University.

HARMON, P; KING, D. 1985. Expert systems. Artificial intelligence in business. Edited by Jobn Wiley \& Sons. New York, E.E.U.U.. WILEY. 283 p.

HILJE Q., L.; CASTILLO M.. L.E.; THRUPP, L.A.; WESSELING H.; 1. 1987 El uso de los plaguicidas en Costa Rica. San José. C.R. UNED: Heliconia. 150 p.

HORN. D.J. 1988. Ecological approach topest management. Edited by David Pimentel. New York, E.E.U.U. The Guilford Press. $285 \mathrm{p}$.

KING, A.B.S.; SAUNDERS. J.L. 1979. Las plagas invertebradas de los cultivos anuales alimenticios en América Central. Londres. G.B. ODA. 182 p.

MAGIFAO/PNUD (NIC.) 1976. Control integrado de plagas de maíz. sorgo y frijol. Managua, NIC., Proyecto Control Integrado de Plagas. 58 p.

McKINION. I.M.; LEMMON. H.E. 1985. Expert system for agriculture. Computers and Electronics in Agriculture (E.E.U.U.) 1 (1): 31-40.

NAEGELE, J.A.; COULSON. R.N.; STONE. N.D.; FRISBIE. R.E. 1985. The use of expert system to integrate and deliver IPM technology. pp. 692-711. In Frisbie. R.C. y Adkinsson, P.L. (eds). integrated pest management on major agricultural systems. Texas A \& M University.

PARSA YE, K.; CHIGNELL, M. 1988. Expert system for experts. Edited by John Wiley \& Sons. New York. E.E.U.U., WILEY. 461. p.

REYES C., L.; HERNANDEZD.. A. 1987. Sistema de referencia entomológico computarizado. In Congreso de manejo integrado de plagas (4, 1987, memorias). Guatemala (G.U.). pp. 441-447.

SAUNDERS. J..L.; KING, A.B.S.; VARGAS S., C.L. 1983. Plagas de los cultivos en América Central. Una lista de referencia. Turrialba. C.R. Centro Agronómico Tropical de Investigación y Enseñanza. (Serie Técnica. Boletín técnico / Centro Agronómico Tropical de Investigación y Enseñanza; No. 9) 92 p.

WISIOL I., K.; H. HESKETH. J.D. 1987. Plant growth modeling for recure management. Florida. E.E.U.U. CRC. vol. II 170 p. 